\section{Blau-Schwarz-Regel verbessert Diagnose von nodulären Melanomen}

Da noduläre Melanome nicht die klinischen $A B C D-K r i t e r i e n$ erfüllen, ist die Diagnose oft schwierig. Mithilfe einer einfachen Regel, bei der auf blaue und schwarze Pigmentflächen geachtet wird, lassen sich solche Tumoren per Dermatoskopie mit hoher Sensitivität erkennen.

D as tückische an nodulären Melanomen ist bekanntlich, dass sie klinisch oft nur schwer von Hämangiomen, pyogenen Granulomen, seborrhoischen Keratosen oder anderen pigmentierten Nävi unterscheidbar sind. Dermatologen um Dr. Guiseppe Argenziano aus Reggio nell'Emilia in Italien haben nun versucht, solche malignen Melanome mithilfe einer simplen Regel durch die Dermatoskopie besser zu erkennen. Bei dieser BlauSchwarz-Regel wird darauf geachtet, ob in pigmentierten nodulären Läsionen eine Kombination von blauen und schwarzen Pigmentflächen sichtbar ist, die mindestens $10 \%$ der Oberfläche bedecken. Die Pigmentierung darf dabei jedoch nicht komedoähnlich wie bei seborrhoischer Keratose oder lakunär wie bei Hämangiomen auftreten.

Die Dermatologen prüften 283 verdächtige pigmentierte noduläre Läsionen per Dermatoskopie mit der Blau-SchwarzRegel und nach Standardkritieren für Melanome. Zu diesen Kriterien zählten sie irreguläre Streifen, Regressionsstrukturen, irreguläre braune Globuli und irreguläre strukturlose Flächen sowie atypische Pigmentnetze. Trat wenigstens eines dieser Merkmale auf, galten die Standardkriterien als erfüllt. Anschließend wurde die Diagnose histopathologisch gesichert und mit den Dermatoskopie-Ergebnissen verglichen.
Insgesamt erwiesen sich dabei $160 \mathrm{der}$ Läsionen als bösartig, 78 davon waren Melanome (28\%), 75 Basalzellkarzinome (27\%). Von den Melanomen wurden 61 (78\%) mit der Blau-Schwarz-Regel richtig erkannt, aber nur 34 (47\%) anhand der Standardkriterien. Wurden beide Verfahren kombiniert, so ließen sich 66 richtig erkennen, was eine Sensitivität von $86 \%$ entspricht. Auf der anderen Seite erwiesen sich mit der Blau-Schwarz-Regel 40 von 205 Befunden als falsch positiv - hier lag kein Melanom vor, mit Standardkriterien ergaben sich dagegen nur 5 von 205.

Fazit: Insgesamt lassen sich mit der BlauSchwarz-Regel in Kombination mit den Standardkriterien die meisten Melanome zuverlässig erkennen, wenngleich dabei eine erhöhte Rate falsch positiver Befunde in Kauf genommen werden muss.

Thomas Müller

Argenziano $\mathrm{G}$ et al. Blue-black rule: a simple dermoscopic clue to recognize pigmented nodular melanoma. Br J Dermatol 2011; 65: 1251-5,

\title{
Hautkrebsbiopsie hat freie Ränder: Weiterschneiden?
}

Ist nach einer Biopsie verdächtiger Hautveränderungen die Diagnose eines spinozellulären oder Basalzellkarzinoms gesichert, folgt eigentlich die Exzision. Doch was ist, wenn der Pathologe meldet: „Biopsieränder tumorfrei“? In manchen Fällen, so das Ergebnis einer kleinen Studie, könnte man es damit vermutlich gut sein lassen.

$\mathrm{D}$ ermatologen um Julie Jackson von der University of California in Los Angeles haben in einer retrospektiven Studie überprüft, was tumorfreie Ränder diagnostischer Biopsate über das Vorhandensein von Resttumorgewebe aussagen. Ihre Stichprobe umfasste 235 Biopsien von weißem Hautkrebs (148 spinozelluläre und 87 Basalzellkarzinome). Zwölf Gewebeproben spinozellulärer Karzinome wiesen freie Wundränder auf, ebenso neun der Basaliombiopsien.

Bei den Spinaliomproben mit freiem Rand handelte es sich um gut differenzierte Karzinome, um Karzinome vom Keratoakanthomtyp und ein Carcinoma in situ. Hier waren nach der folgenden Operation auch alle Exzidate tumorfrei. Ganz anders die Situation bei den Basaliomen: In sieben der neun Fälle, die negative Biospieränder aufgewiesen hatten, fand sich nach der ausgedehnteren Exzision noch Tumorgewebe.

Fazit: „Handelt es sich um gut differenzierte spinozelluläre Karzinome oder solche vom Keratoakanthomtyp, kann man die diagnostische Biopsie mit negativen Rändern vermutlich bereits als Therapie betrachten", schreiben Jackson und ihre Kollegen in ihrem Fazit. Allerdings sei zu berücksichtigen, dass die Zahl von 21 untersuchten Proben mit negativem Rand

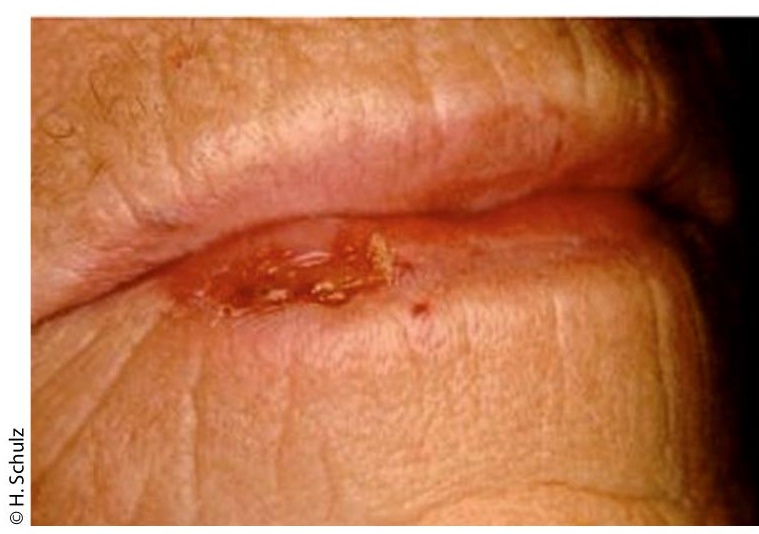

Spinozelluläres Karzinom

recht klein gewesen sei. Bevor aber nicht die Ergebnisse einer größeren prospektiven Studie mit fünfjährigem Follow-up vorlägen, sollte man die derzeitige Therapiestrategie nicht ändern.

Dr. Robert Bublak

Jackson JE et al. Predictive value of margins in diagnostic biopsies of nonmelanoma skin cancers. J Am Acad Dermatol 2011; Nov 14, [Epub ahead of print] 